\title{
The analysis of Caesarean Section Rate based on 10 groups Robson's Classification
}

Lorena Zijaj

Resident at Obstetric- Gynecology Department, Faculty of Medicine, University of Medicine, Tirana, Albania.

Arian Shtylla Obstetric -Gynecologist Doctor at University Hospital of obstetric Gynecology "Koco Gliozheni", Tirana, Albania.

Jola Kerpaci

Resident at Obstetric -Gynecology Department, Faculty of Medicine, University of Medicine, Tirana, Albania.

Irida Dajti

Resident at Obstetric- Gynecology Department, Faculty of Medicine, University of Medicine, Tirana, Albania.

\section{Abstract}

Introduction: Caesarean Section use is growing in a progressive rate globally. The trend in Albania follows the same pattern with the overall rate rising steadily. Specifically, in "Koco Gliozheni" Hospital the data went up from 29.5\% in 2010 to $43.41 \%$ in 2020. The World Health Organization recommends the Robson Classification as an effective way of monitoring and analyzing caesarean section .

Objective: The aim of this article is to evaluate the cesarean section rate at UHOG "Koco Gliozheni".

Material and method: This is a retrospective cross sectional study conducted at UHOG "Koco Gliozheni" in Tirana from January 2016 to May 2017.

Results: 1918 out of 4838 births were performed by caesarean section, which corresponds to a rate of $39.6 \%$. According to Robson classification the largest group was the group 5 with a relative contribution of $31.6 \%$. On second and third place were group 1 and 2 with relative contribution of $22.5 \%$ and $16.7 \%$ respectively. Groups 1 ; 
2; 5 made an account of relative contribution of $76.2 \%$. All other groups had altogether a contribution of $23.8 \%$.

Conclusions: In our study, Robson Groups 5, 1, 2 were identified as the main contributors to the overall CS rate at the UHOG "Koco Gliozheni". It is important that efforts to reduce the overall CS rate should mainly be focused on the primary CS rate (group 1 and 2) and on increasing vaginal birth trail after CS (group 5).

Keywords: Caesarean Section Rate, Vaginal Birth, Robson Classification

\section{Introduction}

Caesarean Section use is growing at a progressive rate globally, accounting for $21 \%$ births worldwide in 2015 from 12\% in 2000 (Boerma, Ronsmans , \& Melesse, 2018). According to

recommendation made for proper prenatal and births care, from a Joint Conference Appropriate Technology for Birth, organized by World Health

Organization in 1985, there is no justification in any specific geographic region to have more than 10-15\% Caesarean Section births (WHO, Appropriate technology for birth, 1985). This rising rate is a crucial public health problem, thus causing debatable questions due to the potential risks both maternal and perinatal.

Figure 1:Trend of Caesarean Sections and vaginal births in "Koco Gliozheni" Hospital during 2010-2020.

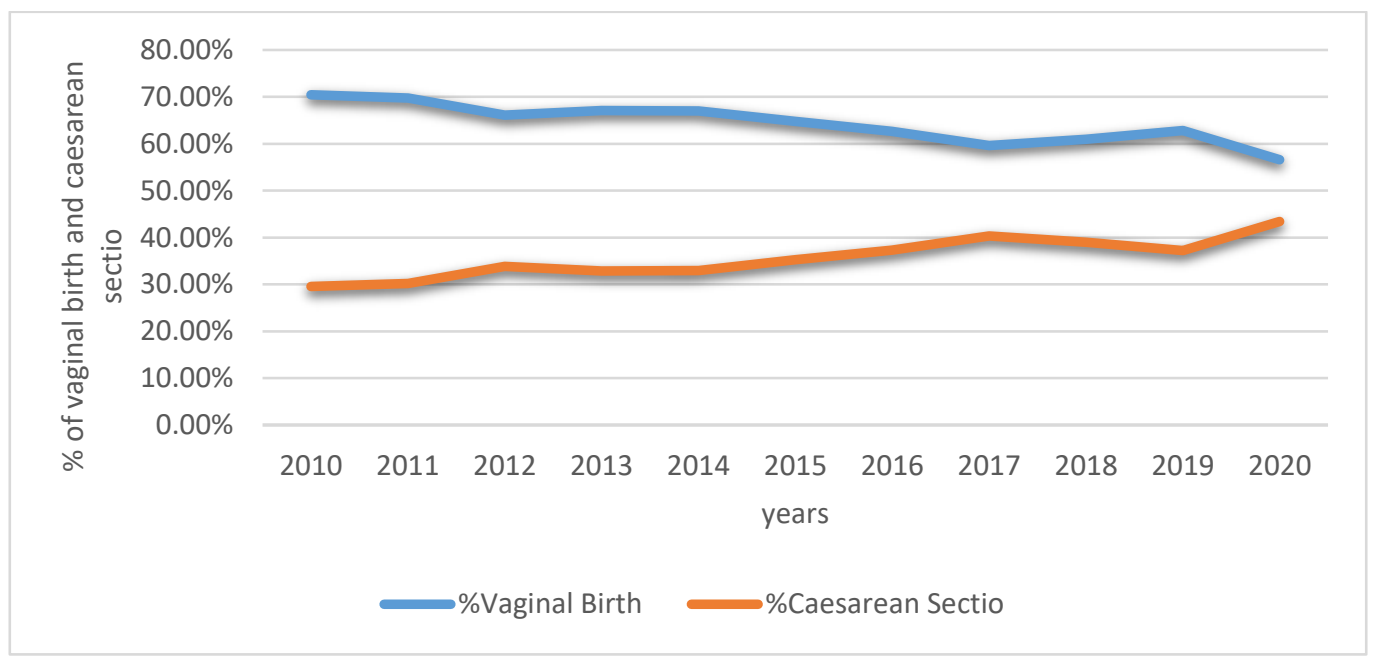


Additionally cost issues and accessibility have promptly become a concern. Statistically across the US overall CS rate was 32\% in 2017 (Cunningham, 2018) compared to approximately $20 \%$ in 1996. In UK CS deliveries have increased $19.7 \%$ of birth in 2000 to $26.2 \%$ in 2015 (Boerma, Ronsmans , \& Melesse, 2018). According to Lancet in at least 15 countries the CS rate exceeds 40\%, including Brazil, 55.5\% Turkey (Bazelian, 2007) and Egypt 53.1\% (Jadoon, 2019). Kosovo, a country with the same ethical traits as Albania is experiencing a swift growth in CS rate. From 2000 to 2015 the figures have increased from 7.5\% to 27.3\%. The trend in Albania follows the same pattern with the overall rate rising steadily. More specifically in "Koco Gliozheni" Hospital the rate has fluctuated during the years. Firstly it climbed from $29.5 \%$ in 2010 to $40.35 \%$ in 2017 , then it went down to $37.2 \%$ in 2019 , jumping to $43.41 \%$ in 2020 . One of the main factors that may have contributed to the sharp rising trend in 2020 is Covid -19 , whose adverse effects in maternal health play a crucial role (Figure 1). In order to understand the drivers of this trend, different authors have created and proposed a consistent and standardized classification known as Robson Classification. Many countries use this randomly in their studies , while other like Albania have not implemented it yet. According to WHO the Robson's Classification is for "all women" who delivered at a specific setting and not only for the women who delivered by Caesarean Section (WHO, Robson classification: implemention manual World Health Organisation, 2017). The groups of the Robson classification include variables: Parity, Previous Caesarean Section, Onset of labor, Number of foetus, Gestational age, foetal lie and presentation (Robson, 2001).

\section{Objectives}

The main objective of this paper is to report an analysis of the CS rate in our hospital "Koco Gliozheni" using the 10 group Robson Classification and to determine trends on a time period from 2016-2017.

\section{Materials and Methods}

This is a retrospective cross-sectional study at the obstetric department of the University Hospital of Obstetric and Gynecology “Koco Gliozheni|"from January 2016 to May 2017. The UHOG "Koco Gliozheni" is a tertiary University hospital supported by 24 hours obstetrics team, pediatric services, anesthetic and neonatal department. This study includes all women who gave births to alive or still born baby of at least 28 weeks gestational age during the above mentioned time period. We have used this cut off for definition of birth ,because the threshold of viability in many low-income countries is birth weight $\geq 1000 \mathrm{~g}$ and gestational age $\geq 28$ weeks. The obtained data were maternal age, parity, gestational age, onset of labor, foetus presentation, 
previous deliveries and previous CS. The data were categorized into 10 groups according to the Robson Classification

System (table 1). The groups are as below: Group 1 Nulliparous woman, single cephalic presentation in spontaneous labor. Group 2 is divided in two subgroups: $2 \mathrm{a}$ Nulliparous single cephalic presentation in spontaneous labor, 2b Nulliparous single cephalic presentation planned caesarean delivery. 3 Multiparous women without uterine scar, single, cephalic, term pregnancy in spontaneous labor. Group 4 is divided in two subgroups: 4a Multiparous women without uterine scar, cephalic, term, pregnancy in induced labor and $4 \mathrm{~b}$ Multiparous women without uterine scar, cephalic term pregnancy, planned cesarean delivery. Group 5 Multiparous with scarred uterus, single, cephalic, term pregnancy. Group 6 Nulliparous single, breech pregnancy. Group 7 Multiparous single breech pregnancy (included women with scarred uterus). Group 8 All women with multiple pregnancy (include them with scarred uterus). Group 9 All women with single or oblique pregnancy (include them with scarred uterus). Group 10 All women with single cephalic preterm pregnancy (include them with scarred uterus). Those variables needed to be analyzed for our study were parity, gestational age, foetal presentation, and previous caesarean section. Statistical analysis and graphics presentation were performed using EXEL 2010 and Microsoft office programs. All missing data cases were excluded from the study.

\section{Results}

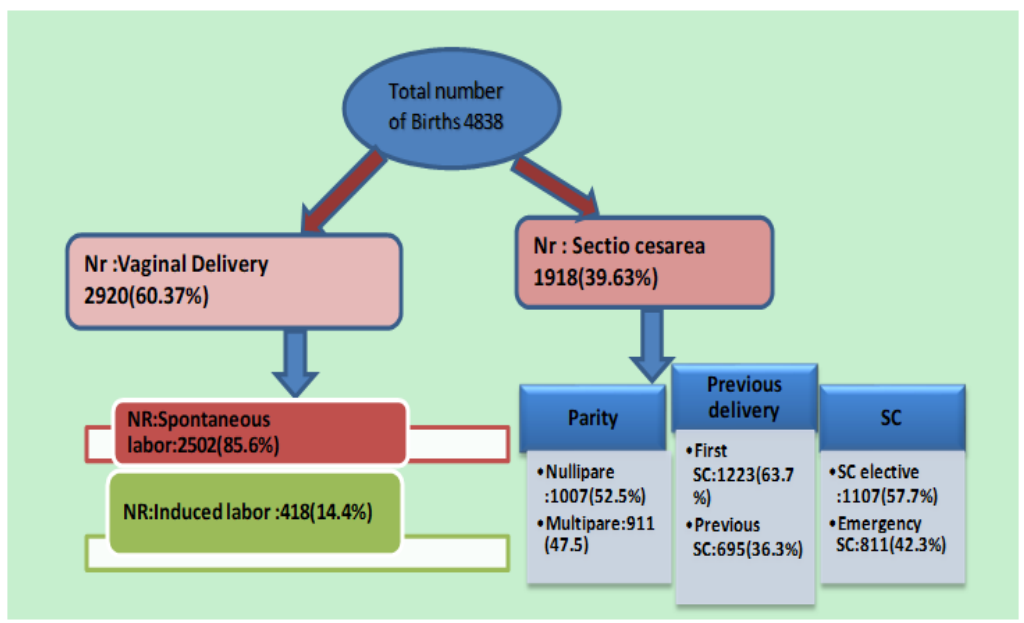

Figure 2:Distribution of Caesarean Sections and vaginal births 
During our study period from January 2016 to May 2017, 4838 women gave birth at University Hospital of Obstetrics-Gynecology “Koco Gliozheni”, Tirana:

1. Nulliparous; single cephalic term pregnancy; spontaneous Labour

2. a. Nulliparous; single cephalic term pregnancy; induced labour

2. b. Nulliparous; single cephalic term pregnancy; planned caesarean delivery

3. Multiparous without uterine scar; single cephalic term pregnancy; spontaneous labour

4. a. Multiparous without uterine scar; single cephalic term pregnancy; induced labour

4. b. Multiparous without uterine scar; single cephalic term, planned caesarean delivery

5. Multiparous with scarred uterus; Single cephalic term pregnancy

6. Nulliparous; single breech pregnancy

7. Multiparous; single breech pregnancy (including women with scarred uterus)

8. All women with multiple pregnancy (including women with scarred uterus)

9. All women with a single oblique or transverse pregnancy (including women with scarred uterus)

10. All women with single cephalic preterm pregnancy (including women with scarred uterus)

Table 1:The ten group Robson's Classification

There were 2388 nulliparous (49.38\%) and 2450 multiparous (50.65\%). CS was performed in 1918 women resulting in an overall CS rate of (39.63\%). The number of CS performed to all

nulliparous and multiparous during this period of time was 1005 (52.5\%) and 911 (47.5\%) respectively. The rate of elective CS was $57.7 \%$ while that emergency CS was 42.3\%, (Figure 2). We attempted to categorize CS according to Robson Classification and rates of each group were demonstrated separately (Table 2). The largest contributors to the overall CS rate were women with previous CS (group 5, multiparous with scarred uterus ) $12.45 \%$. CS rate within this group was $90.6 \%$ (602 out of 664 women). 456 out of 664 women (68.67\%), had performed a CS prior to the onset labor (elective CS). 148 (31.33\%) of women of this group, had attempted a VBAC ( vaginal birth after Cesarean Section). A significant number of these 
multiparous, with at least one previous vaginal birth. The second highest contributors were women included in group 1 (singletons nulliparous, cephalic presentation, at term, spontaneous onset of labor ), with an overall CS rate $8.9 \%$ and with relative percentage of $22.5 \%$.

The group 2 (nulliparous with single cephalic,

full term pregnancy, included labor or pre labor CS) had the third contribution with $6.6 \%$ of overall CS rate and a relative contribution of only $16.7 \%$. If we analyze the group 2 , we find that the $2 \mathrm{~b}$ subgroup (elective caesarean delivery) had a contribute with a $6.3 \%$ of overall CSR and with a relative contribution of $16.3 \%$.

\section{Discussion}

Over the last decades, there has been a progressive increase in the rate of deliveries by caesarean section (CS) in most countries but the drivers for this trend are not completely understood (BetránAP, 2016). Health Organization (WHO) conducted a systematic review that identified 27 different systems to classify CS. These classifications looked at "who" (woman-based), "why" (indication-based), "when" (urgency-based), as well as "where", "how" and "by whom" a CS was performed (Torloni MR, 2011). This review concluded that women-based classifications in general, and the 10 Groups classification in particular (MS, 2001), were in the best position to fulfill current international and local needs. The 10 Groups classification (also known as the "TGCS Ten Groups Classification System" or the "Robson Classification") was created to prospectively identify well-defined, clinically relevant groups of women admitted for delivery and to investigate differences in CS rates within these relatively homogeneous groups of women (MS, 2001). WHO expects that the use of the Robson Classification will help health care facilities to: $\bullet$ Identify and analyze the groups of women which contribute most and least to overall CS rates. $\bullet$ Compare practice in these groups of women with other units who have more desirable results and consider changes in practice. - Assess the effectiveness of strategies or interventions

targeted at optimizing the use of CS. - Assess the quality of care and of clinical management practices by analyzing outcomes by groups of women. - Assess the quality of the data collected and raise staff awareness about the importance of this data, interpretation and use (https://creativecommons.org/licenses/by-ncsa/3.0/igo)., 2007).

Our study reports the data from low income countries like Albania. During the study from January 2016 to May 2017, gave birth 4828 women,1918 (39.63\%) out of 4839 
performed CS. The rate is higher than developed countries like France (31\%), Australia (28\%), USA (31.1\%) and lower than Egypt (54\%), Turkey (51.9\%), South America (42,9\%). The main contributor to the overall CS rate were group 5, 1, 2. Group 5: (multiparous with prior caesarean section, singleton, $>37$ weeks): provides the highest contribution with $31.6 \%$ of all CS and with $90.6 \%$ CS rate of women in this group much higher than Robson's references (50-60\%). On further analysis we conclude that $68.67 \%$ of women in this group had an elective CS and only $31.33 \%$ of women had attempted trail of labor after CS (TOLAC), even though VABC has a success of 75\% (Williams, 2018). In some studies in low income countries, success of VBAC is as low as $27.4 \%$ to $53.6 \%$ (Agrawal, 2007). But on the other hand, countries with high socio- economic status, reported a higher CS rate from group 5 (multiparous with prior caesarean section, singleton, $>37$ weeks) respectively $61 \%$ and $47 \%$ (Zhang, 2016).

\begin{tabular}{|c|c|c|c|c|c|c|}
\hline Group nr & $\begin{array}{l}\text { Total nr of } \\
\text { women } \\
\text { delivered in } \\
\text { each group(N) }\end{array}$ & $\begin{array}{l}\text { Total nr of } \\
\text { CS in each } \\
\text { group(n) }\end{array}$ & $\begin{array}{l}\text { Group } \\
\text { size }(\%)\end{array}$ & $\begin{array}{l}\text { Group } \\
\operatorname{CSR}(\%)\end{array}$ & $\begin{array}{l}\text { Absolute } \\
\text { group } \\
\text { contribution to } \\
\text { overall CS } \\
\text { rate }(\%)\end{array}$ & $\begin{array}{l}\text { Relative } \\
\text { contribution of } \\
\text { the group to } \\
\text { overall CSR } \\
(1978 \mathrm{CS}) \%\end{array}$ \\
\hline 1 & 1507 & 432 & 30.3 & 28.6 & 8.9 & 22.5 \\
\hline 2 & 550 & 321 & 11 & 58.3 & 6.6 & 16.7 \\
\hline 3 & 1309 & 86 & 27 & 6.5 & 1.8 & 4.5 \\
\hline 4 & 227 & 57 & 4.6 & 25 & 1.2 & 2.9 \\
\hline 5 & 664 & 602 & 13.7 & 90.6 & 12.45 & 31.6 \\
\hline 6 & 135 & 128 & 2.7 & 94.8 & 2.6 & 6.7 \\
\hline 7 & 79 & 66 & 1.6 & 83.5 & 1.4 & 3.4 \\
\hline 8 & 90 & 78 & 1.8 & 86.6 & 1.6 & 4 \\
\hline 9 & 23 & 23 & 0.47 & 100 & 0.48 & 1.2 \\
\hline 10 & 254 & 125 & 5.2 & 49.2 & 2.6 & 6.5 \\
\hline Total Number & 4838 & 1918 & $100 \%$ & $39.63 \%$ & $39.63 \%$ & $100 \%$ \\
\hline
\end{tabular}

Table 2:Robson report table for University Hospital Center Obsteric-Gyneocology "Koco Gliozheni"

Group size (\%) =n of women in the group.total number $\mathrm{N}$ women delivered in the hospital*100

Group CS rate $(\%)=n$ of CS in the group/total $N$ of women in group*100

Absolute contribution $(\%)=\mathrm{n}$ of $\mathrm{CS}$ in group /total $\mathrm{N}$ of women delvered in hospital*100

Relative contribution (\%) $=\mathrm{n}$ of CS in group/total $\mathrm{N}$ of CS in hospital*100 


\section{CS (Caesaren Section)}

Color signifies the high-risk group

Some of the factors that contribute to the decreasing of percentage of VBAC are myths such as "Once a caesarean always a caesarean", lack of training and malpractice. Uterine rupture is a possible complication during vaginal birth with a scarring uterus, but studies calculate the risk from $0.2 \%$ to $0.8 \%$ (ACOG, 2010). Group 1: (CS performed during labor) contributes with approximately $22.5 \%$ of CSR, and $28.6 \%$ within the group. Referring to Robson's finding, this group should account for no more than 10\% (WHO, Robson classification: Implemention Manual, 2017). The high rate of CS in our Hospital can be explained by the lack of infrastructure. For instance, the well-being of foetus during labor has been monitored by intermittent CTG and sometimes only with feotal stethoscope.

Furthermore, reasons like insufficient training of staff for CTG interpretation, lack of other foetal assessment such as foetal scalp blood sampling and cord blood PH play a crucial role. Given that, the doctors have to make a decision based only in CTG findings. Additionally, epidural or any other anesthesia is not performed regularly in our everyday practice. The instrumental deliveries have reached at a critical low point, not only in "Koco Gliozheni" Hospital, but globally. Group 2: (Nulliparous women cephalic at term induced labor or elective CS) has the third highest contribution, with $16.7 \%$ of overall CSR and $58.3 \%$ within the group. Robson's rate references for this group are $20-35 \%$. After a thorough analysis, we conclude that different from references, the subgroup 2a (induced labor) is relatively smaller than $2 \mathrm{~b}$ (elective CS). This can be explained with underreported procedure of labor induction on patient's files. The elective section (2b) is mostly performed for nonmedical indications. The most common reason is the request of the woman as she refuses to try the vaginal birth, due to anxiety, fear of pain, and concerns of pelvic injuries. If we analyze the numbers in the subgroup of elective Caesaren (2b), we conclude that the group contributes with $16.3 \%$ of overall CSR and with $54.4 \%$ within the group. So it is a considerable percentage, compared with the contribution of group 2 itself in total. Group 3-4 (multiparous women at term induced or elective CS) have a relatively low contribution, which reaches all together $7.4 \%$. They are labelled the "low risk group". Groups 6 - 10 were smaller groups with an overall size of $11.77 \%$, and with the highest group CSR that reach up to $100 \%$ in group 9 (all women with single pregnancy with transverse or oblique lie, including women with previous uterine scar). This group are presented with an unavoidable obstetric condition (like 
breech presentation, multiple pregnancy abnormal fetal presentation or premature birth, several maternal obstetrical conditions) that has been served as medical indication for CS. But on the other hand the contribution to the overall CSR is lower and reaches only $32 \%$.

\section{Conclusions}

In our study, Robson's groups 5, 1, 2 were identified as the main contributors to the overall Cesarean Section rate at the "Koco Gliozheni"

University Hospital, Tirana. It is important that the effort to reduce the overall CSR, should mainly be focused on the primary CS rate (group 1 and 2) and increasing the attempt of vaginal birth after cesarean section. We believe that this classification can be incorporated successfully in the routine of obstetrical management, and implemented in the collection of maternal and perinatal data system to improve the evaluation of Caesarean Section rate. We consider that future work should be done to harmonize data between different countries, so more detailed collection systems and protocols are obtained.

Acknowledgments None declared.

Conflict of interest None declared.

\section{References}

[1] ACOG. (2010). Vaginal birth after cesarean delivery. ACOG PRACTICE BULLETIN, 116.

[2] Agrawal, C. (2007). Evalutation of pregnant women with scarred uterus in a low resourse countries. Journal of Obsteric Gynaecology Research, 651.

[3] Bazelian, A. (2007). Health consequences of the increasing caesarean rates. Epidemiology, 485.

[4] BetránAP, Y. J. (2016). The increasing trend in caesarean section rates: regional and national estimates: 1990-2014. 4. PLoSONE., 11(2).

[5] Boerma, T., Ronsmans , C., \& Melesse, D. (2018). Global epidemiology of use and disparieties in caesarean sections . Lancet, 392.

[6] Robson MS. (2001). Classification of caesarean sections. Fetal and Maternal Medicine Review., 12(1):23-39.

[7] Chinnock. (2007). Obstetric trainees'experience in vaginal birth delivery. Obstet Gynecol, 110.

[8] Cunningham, K. (2018). Williams Obsterics. In Williams, Cesarean delivery and peripartum hysterectomy (p. 591). USA. 
[9] https://creativecommons.org/licenses/by-nc-sa/3.0/igo)., W. (2007). Robson Classification: Implementation Manual. Geneva:.

[10] Jadoon. (2019). Analysis of Caesarean Section based on Robson group's classification at Behna University Hospital . Woman Birth, 328.

[11] Jasenko, I. (2016). Caesarean Sectio rate Analysis in Universiy hospital Tuzla -according to Robson's classification.

[12] Robson. (2001). Classification of caesarean section. Medicine Review, 39.

[13] Torloni MR, B. S. (2011). Classifications for cesarean section: a systemativ review . PLoSONE, 6(1):e1456.

[14] WHO. (1985). Appropriate technology for birth. Lancet, 436.

[15] WHO. (2017). Robson classificatio: implemention manual World Health Organization .

[16] Williams. (2018). Delivery after Cesarean section. In William, Obstetrics (p. 591). USA.

[17] Zhang, G. ,. (2016). A cesarean Cesction in subgroups of women and perinatal outcomes. BJOG, 73. 